\title{
ADAPTAÇÕES DO MÚSCULO ESQUELÉTICO AO ENVELHECIMENTO E AO TREINAMENTO DE FORÇA: UMA REVISÃO NARRATIVA SOBRE A SARCOPENIA E A DINAPENIA
}

\author{
Rochelle Rocha Costa ${ }^{1}$ \\ Thaís Reichert² \\ Luiz Fernando Martins Kruel ${ }^{3}$
}

resumo

O crescimento da população idosa ressalta a importância da investigação sobre os efeitos do envelhecimento no sistema musculoesquelético. O objetivo desse estudo foi discutir sobre a terminologia da sarcopenia e da dinapenia, bem como revisar as adaptações do músculo esquelético ao envelhecimento e ao treinamento de força.

1 Graduada em Educação Física (Licenciatura plena) pela Universidade Federal do Rio Grande do Sul (UFRGS). Doutora em Ciências do Movimento Humano (UFRGS). Coordenadora Adjunta do Grupo de Pesquisas em Atividades Aquáticas e Terrestres (UFRGS - Departamento de Educação Física). E-mail: rochelle.costa@ufrgs.br.

2 Graduada em Educação Física (Licenciatura) pela UFRGS. Mestre em Ciências do Movimento Humano (UFRGS). Pesquisadora do Grupo de Pesquisas em Atividades Aquáticas e Terrestres (UFRGS - Departamento de Educação Física). E-mail: thais_reichert@hotmail.com.

3 Graduado em Educação Física (Licenciatura plena) pela UFRGS. Docente (professor titular) no Departamento de Educação Física da Escola de Educação Física, Fisioterapia e Dança da UFRGS. Coordenador do Grupo de Pesquisas em Atividades Aquáticas e Terrestres (UFRGS - Departamento de Educação Física). E-mail: kruel@esef.ufrgs.br. 
A busca eletrônica foi conduzida nas bases de dados MEDLINE e Scopus em março de 2016 utilizando-se os termos aging, skeletal muscle, strength e dynapenia. O declínio da capacidade de produção de força muscular em decorrência do envelhecimento recebeu a denominação científico-clínica de Dinapenia. Apesar disto, ainda existe uma gama de pesquisadores que utiliza esta definição como parte do construto de Sarcopenia. Fatores neurais e fatores musculares constituem os mecanismos que contribuem para a instalação da dinapenia. Os fatores neurais englobam alterações no recrutamento e na sincronização de unidades motoras, a queda taxa de disparo e na velocidade de condução nervosa, a denervação das fibras musculares tipo II (rápidas) por morte neuronal e reinervação das fibras tipo I (lentas). Os fatores musculares são constituídos por alterações na arquitetura muscular, mudanças de tipo de fibra, disfunções no processo de acoplamento excitação-contração, alterações estruturais na miosina e infiltrações de lipídios intra e intermusculares. A dinapenia é diagnosticada baseada em uma avaliação dos fatores de risco, do teste de força de extensão de joelhos ou preensão manual. A partir do entendimento dos diversos mecanismos que contribuem para a instalação da dinapenia, pesquisadores das ciências da saúde podem ter embasamento para uma melhor prescrição de tratamentos preventivos ou mesmo de reabilitação da dinapenia.

palavras-chave

Músculo Esquelético. Envelhecimento. Força Muscular. Debilidade Muscular.

\section{Introdução}

Nas últimas décadas, a população idosa tem apresentado um crescimento exponencial (OMS, 2015). Atualmente, os idosos já representam 12,3\% da população mundial total e projeções indicam que, no ano de 2050, eles passarão a representar 21\%, superando inclusive o número de jovens (OMS, 2015). No Brasil, é esperado que o número de idosos triplique até a metade do século, revelando um crescimento acima do nível mundial e atingindo a representação de 30\% da população brasileira (IBGE, 2010).

O processo de envelhecimento é acompanhado por uma série de alterações no organismo, dentre as quais destaca-se o declínio de uma das suas 
principais funcionalidades: a capacidade de produzir força (GOODPASTER et al., 2006; MANINI; CLARK, 2012; TIELAND; TROUWBORST; CLARK, 2018). Quando esta função encontra-se demasiadamente afetada, pode ocasionar uma perda incapacitante da independência e da mobilidade do idoso, resultando em uma necessidade de cuidados especializados de terceiros ou mesmo de instituições especializadas. O baixo nível de força muscular também tem sido identificado como um preditor independente de hospitalização, incapacidade e, até mesmo, morte (ALEXANDRE et al., 2014).

As graves consequências da redução da força muscular associada ao envelhecimento demonstram a relevância de investigações acerca deste processo. Nesse sentido, considerando o crescimento da população idosa, diversos estudos têm objetivado identificar as adaptações do músculo esquelético ao avanço da idade. A compreensão dos processos e dos mecanismos envolvidos favorece a implementação de terapias preventivas e de tratamento, como, por exemplo, aquelas implementadas por meio do treinamento físico. Embora a literatura existente esclareça diversos pontos isolados dessas questões, nenhum estudo foi encontrado reunindo as evidências e atualizando o conhecimento sobre as terminologias e o diagnóstico da sarcopenia e da dinapenia, a dissociação da relação entre a força e a massa muscular, os fatores determinantes no declínio da força durante o envelhecimento, e os efeitos do treinamento de força sobre a força e a massa muscular de idosos. Sendo assim, justifica-se a publicação de um estudo de revisão narrativa, em língua portuguesa, sobre o tema, gerando maior visibilidade e alcance destas evidências à comunidade científica em geral. Dessa forma, o objetivo do presente estudo foi discutir sobre a terminologia da sarcopenia e da dinapenia, bem como, revisar as adaptações do músculo esquelético ao envelhecimento e ao treinamento de força.

\section{Design do estudo e procedimentos}

Este trabalho caracteriza-se como uma revisão narrativa apresentada em caráter descritivo-discursivo, na qual são apresentados e discutidos tópicos relacionados à dinapenia. A pesquisa foi realizada em julho de 2019 e a busca eletrônica foi conduzida utilizando-se as bases de dados MEDLINE (por meio do PubMed) e Scopus. Os termos descritores escolhidos foram aging, skeletal muscle, strength e dynapenia, bem como suas combinações. Estes descritores foram associados a seus termos $\mathrm{MeSH}$ correspondentes. Foram selecionados estudos apresentados nas línguas inglês e português, não havendo restrição quanto à data de publicação, que trouxessem informações relevantes acerca das 
adaptações do músculo esquelético ao processo de envelhecimento, sendo elas relacionadas ao comportamento da massa muscular e/ou da força muscular, de ordem funcional ou mecanística. Além disso, foram selecionados os estudos que tratassem da relação entre essas variáveis e das terminologias utilizadas na literatura científica sobre esse tema. Adicionalmente, foram consultados estudos citados nas listas de referências dos artigos encontrados na busca eletrônica, selecionados para esta revisão.

\section{Sarcopenia e dinapenia: definições}

A definição do termo sarcopenia, para utilização na área da saúde, data de 1989, tendo sido sugerida pelo Dr. Irwin Rosenberg, preocupado com o declínio da funcionalidade advindo da redução da massa muscular corporal decorrente do envelhecimento (ROSENBERG, 1989). Ele propôs o termo sarcopenia (do grego: pobreza de carne), que foi originalmente definido como a redução da massa muscular relacionada à idade, na ausência de doenças (EVANS, 1995). Embora a proposta inicial do termo sarcopenia estivesse relacionada somente à redução de massa muscular, com o passar dos anos foi agregando sentidos e passando a designar a perda de massa muscular e também a redução na força relacionados ao envelhecimento (NARICI; MAFFULLI, 2010). Um exemplo disto está na definição proposta pelo Grupo Europeu de Trabalho em Sarcopenia com o Idoso da Sociedade Geriátrica Britânica, em sua publicação de 2010 que define sarcopenia como "uma síndrome caracterizada pela perda generalizada e progressiva de massa muscular esquelética e força, com risco de consequências adversas, como incapacidade física, pobre qualidade de vida e morte" (CRUZ-JENTOFT et al., 2010, p. 413, tradução nossa).

Entretanto, a vinculação na mesma palavra (sarcopenia) das alterações que ocorrem na massa muscular e na força, em decorrência do envelhecimento, implica em aceitarmos a existência de uma relação direta entre as duas variáveis, ou seja, que quando houver alteração na quantidade de massa muscular de um indivíduo podemos esperar como resposta uma mudança na capacidade de produção de força (CLARK; MANINI, 2008). Contudo, a literatura atualmente é farta em estudos que demonstram que outros fatores, além da massa muscular, atuam na regulação da capacidade de produção de força e que este não é o principal mecanismo de explicação para suas alterações, conforme será discutido nos parágrafos a seguir.

Estudos confirmaram a dissociação da linearidade entre a massa muscular e a força, demonstrando níveis não significativos de contribuição da primeira 
para o desenvolvimento da segunda, embora ambas estejam associadas a um maior declínio funcional e à morte precoce (NEWMAN et al., 2006; VISSER et al., 2005). Consequentemente, em 2008 vislumbrando a necessidade da separação dos conceitos de redução da massa muscular e da força como consequência do envelhecimento em duas nomenclaturas distintas, é sugerido que o termo sarcopenia limite-se à sua definição original e propõem-se a nomenclatura dinapenia para designar a diminuição nos níveis de força que ocorrem em decorrência do envelhecimento (CLARK; MANINI, 2008). Do grego, dinapenia pode ser traduzido como "pobreza de força", sendo, portanto, um termo bastante adequado considerando a sua definição.

\section{Dissociação da relação "força e massa muscular"}

As primeiras investigações acerca da contribuição da massa muscular para a capacidade de produção de força reportam que a primeira explicaria $35 \%$ da segunda, tanto em pessoas jovens quanto em idosos, no entanto estes estudos são de corte transversal (FRONTERA et al., 1991; MAUGHAN; WATSON; WEIR, 1983). Em contrapartida, trabalhos de corte longitudinal, têm demonstrado que as alterações advindas do declínio da massa muscular explicam no máximo 5\% da diminuição total da força (HUGHES et al., 2001) e que as alterações nestas variáveis apresentam uma associação fraca tanto em homens $(r=0.24)$ quanto em mulheres $(r=0.28)$ (GOODPASTER et al., 2006). Ainda, esses estudos evidenciaram que a manutenção ou mesmo o ganho de massa muscular não previnem a queda na força que ocorre em decorrência do envelhecimento.

Em um acompanhamento de 120 pessoas com idades entre 46 e 78 anos, durante 10 anos, foi observado que a queda na força muscular de extensores de joelhos apresentou uma correlação fraca $(r=0.21)$ com a diminuição na massa muscular (HUGHES et al., 2001). Estes achados concordam com os observados em 1880 idosos acompanhados durante 3 anos (GOODPASTER et al., 2006), nos quais evidenciou-se uma queda anual 3 vezes maior na força de extensores de joelhos quando comparada a diminuição observada na massa livre de gordura da coxa. Em conjunto, esses dados indicam uma dissociação entre a massa muscular e a força, conduzindo à noção de que outros fatores adaptativos contribuem para regular os níveis de força durante o envelhecimento, como por exemplo, fatores neurais (CLARK, 2019), metabólicos e celulares.

Outra maneira de examinar a dissociação da força e da massa muscular com o envelhecimento pode ser por meio da interpretação dos trabalhos que 
avaliam os modelos de uso reduzido. Verificou-se que após 4 semanas de desuso, ocorre uma redução de maior magnitude na força de quadríceps (15\%) quando comparada à diminuição observada na massa muscular (9\%) e, ainda, que as adaptações nos fatores neurais foram capazes de explicar aproximadamente $50 \%$ da redução da força, enquanto que os fatores musculares explicaram em torno de 40\% (CLARK et al., 2006).

Em suma, parece viável assumir a inexistência de uma relação direta entre massa muscular e capacidade de produção de força no envelhecimento, visto que: os estudos longitudinais com idosos indicam uma dissociação entre a perda de massa muscular e a força; e as alterações na massa muscular e na força resultantes de desuso parecem não seguir o mesmo padrão de comportamento ao longo do tempo. Assim, para uma melhor compreensão dos processos adaptativos do músculo esquelético ao envelhecimento, torna-se necessário, o conhecimento dos fatores envolvidos no declínio da produção de força, que não somente a modulação da quantidade de massa muscular.

\section{Fatores determinantes no declínio da força durante o envelhecimento}

Os elementos que influenciam diretamente no declínio da força muscular podem ser subdivididos em duas amplas categorias: fatores neurais e fatores musculares, ambos com participação ativa no processo de dinapenia. Dentre os fatores neurais podemos destacar alterações no recrutamento e na sincronização de unidades motoras, a queda taxa de disparo, a denervação das fibras musculares tipo II (rápidas) por morte neuronal, e reinervação das fibras tipo I (lentas) e a diminuição da velocidade de condução nervosa (LAURETANI et al., 2006; CLARK, 2019). O presente artigo visa identificar os principais elementos relacionados aos fatores musculares que contribuem para a instalação e evolução da dinapenia. Portanto, este será o foco deste capítulo.

São diversos os fatores musculares pelos quais o músculo tem sua capacidade de produção de força reduzida com o envelhecimento. Nesse contexto, é possível identificar como principais: as mudanças na arquitetura muscular, a redução na velocidade de contração, as alterações estruturais na miosina e a queda na qualidade muscular do idoso.

No que se refere às alterações observadas na arquitetura muscular ao longo do processo de envelhecimento, acredita-se que estas podem ser as maiores preditoras da capacidade de geração de força pelo músculo (LIEBER; FRIDÉN, 2001). Nesse sentido, estudos sugerem que idosos apresentam, em 
geral, um comprimento de fascículo reduzido quando comparados a seus pares jovens (LAURETANI et al., 2006; NARICI; MAGANARIS, 2006). Além disso, foram verificadas diminuições no ângulo de penação, na rigidez tendínea e na densidade muscular no decorrer do envelhecimento (LAURETANI et al., 2006; NARICI; MAGANARIS, 2006; BATISTA; VAZ, 2009). Tais mudanças estão diretamente envolvidas nas alterações das propriedades mecânicas musculares, o que acarreta redução importante na capacidade de produção de força do músculo, em diferentes comprimentos e velocidades, podendo assim influenciar negativamente as atividades de vida diária dos idosos (BATISTA; VAZ, 2009).

O ângulo de penação, ou seja, o ângulo entre a direção das fibras musculares e a linha de geração de força de um músculo, tende a apresentar redução com o passar dos anos de vida e esta queda parece estar associada à diminuição na área de secção transversa do músculo em questão (NARICI et al., 2003). Contudo, tal constatação pode ser dependente da localização do músculo que se trata, sendo este componente dos membros superiores ou inferiores. Embora um maior ângulo de penação do músculo vasto lateral tenha sido encontrado em idosos, não foi evidenciada qualquer diferença no músculo tríceps braquial dos idosos quando comparado ao dos jovens (KUBO et al., 2003). Alguns autores sugerem que este comportamento pode ser explicado pelo maior envolvimento dos músculos dos membros inferiores (com predominância das fibras de contração lenta) nas atividades de vida diária dos idosos, o que pode contribuir para que estes grupamentos musculares sejam mais afetados pelo processo de envelhecimento (BATISTA; VAZ, 2009). De forma similar, estudos têm demonstrado que o envelhecimento está associado a uma redução na rigidez dos tendões, aumentando, dessa forma, sua complacência, tornando-os mais elásticos (KARAMANIDIS; ARAMPATZIS, 2006). Esses fatores, em conjunto resultam em uma menor capacidade de transmissão da força produzida pelos músculos ativos aos ossos e consequentemente às articulações envolvidas no movimento.

Conforme citado anteriormente, outro elemento que contribui de forma importante para a diminuição da capacidade de produção de força muscular em idosos é a densidade muscular diminuída. Músculos esqueléticos de pessoas idosas apresentam menor densidade miofibrilar, assim, apresentando quantidade diminuída de unidades contráteis (BATISTA; VAZ, 2009). Acredita-se que essa adaptação esteja associada a uma reduzida capacidade de sintetizar novas proteínas em idosos (HUNTER; PEREIRA; KEENAN, 2016; TIELAND; TROUWBORST; CLARK, 2018), devido a redução no RNAm responsável pela expressão do gene da proteína miofibrilar (WELLE; BHATT; THORNTON, 2000; TIELAND; TROUWBORST; CLARK, 2018). De fato, indivíduos idosos possuem 
uma taxa de síntese de miosina de cadeia pesada 44\% menor em comparação a indivíduos jovens (HUNTER; PEREIRA; KEENAN, 2016).

Além disso, o envelhecimento parece contribuir para a redução na velocidade de contração do músculo como um todo, podendo esta redução ser atribuída, dentre outros fatores, à transição das fibras rápidas para lentas e/ou à inadequada adaptação no acoplamento excitação-contração (DIMITRU et al., 2018). O fenômeno de acoplamento excitação-contração se refere ao processo fisiológico de conversão do sinal neural para a ativação muscular na contração e consequente geração de força (MACINTOSH; GARDINER; MCCOMAS, 2006). Este envolve diversos eventos complexos, e o rompimento ou desacoplamento em algum ponto desta via de acoplamento excitação-contração resulta em redução da capacidade intrínseca de produção de força e, consequentemente, dinapenia (DIMITRU et al., 2018; TIELAND; TROUWBORST; CLARK, 2018).

Dentre os fatores que podem afetar negativamente o processo de excitação-contração, ressalta-se a importância da disponibilidade de receptores de membrana na célula muscular. Em decorrência do processo de envelhecimento, ocorre uma redução do número de receptores dihidropiridina nos túbulos $\mathrm{T}$ e na membrana do retículo sarcoplasmático (DELBONO, 2011), resultando em um desacoplamento dos canais de liberação de cálcio e falha na transdução dos potenciais de ação como resposta mecânica (JIMENEZ-MORENO et al., 2008; HUNTER; PEREIRA; KEENAN, 2016). Mais recentemente, alguns estudos têm demonstrado que outras proteínas da célula muscular esquelética que estão envolvidas nesse processo possuem contribuição direta na fraqueza muscular associada à idade. Um exemplo é a redução na expressão da proteína retículo-plasmática juncional JP45, que foi observada em decorrência do envelhecimento (ANDERSON et al., 2003; ANDERSON et al., 2006; DELBONO et al., 2007). Esta proteína altera os níveis de expressão das subunidades dos receptores de dihidropiridina, afetando o processo de acoplamento excitação-contração.

Ainda a nível molecular, sugere-se que a função muscular debilitada pelo envelhecimento pode ser resultado de alterações estruturais na miosina, causando mudanças na cinética dos ciclos de pontes cruzadas (LOWE; THOMAS; THOMPSON, 2002). Em um estudo em modelo animal, demonstrou-se que a atividade da enzima miosina ATPase não se altera com o envelhecimento, portanto, atribui-se o declínio da contratilidade muscular às mudanças na estrutura da miosina das fibras dos ratos idosos (LOWE; THOMAS; THOMPSON, 2002). Esta alteração estrutural se refere a uma dissociação das cabeças pesadas de miosina do sítio da actina 30\% maior nas fibras de animais idosos quando comparados às dos jovens. Este fenômeno indica que os menores níveis de força produzidos pelas fibras idosas podem ser atribuídos à migração do 
fluxo das cabeças de miosina de um estado de ligação forte para um estado fraco de ligação durante a contração muscular.

Em estudo com humanos, 1880 idosos foram acompanhados durante 3 anos e observou-se que a magnitude de redução na força é maior do que a concomitante queda na quantidade de massa muscular, sugerindo um declínio na qualidade muscular (GOODPASTER et al., 2006). Neste sentido, infiltrações de lipídios inter e intramusculares são frequentemente observadas com o envelhecimento, este último ocasionando um efeito de redução na densidade muscular (GOODPASTER et al., 2000; GOODPASTER et al., 2001; SONG et al., 2004). Foi demonstrado que este fenômeno pode afetar negativamente a funcionalidade do músculo, conduzindo a uma queda na capacidade de produção de força (GOODPASTER et al., 2000; GOODPASTER et al., 2001). Além disso, maiores infiltrações de gorduras intramusculares, bem como menores níveis de força muscular estão associados a uma maior incidência de limitações de mobilidade em homens e mulheres idosos (VISSER et al., 2005). Somado a estes fatores, a produção de citocinas, pelo tecido adiposo, também tem sido associada a uma baixa capacidade de produção de força muscular (BAUTMANS et al., 2005; DRAGANIDIS et al., 2016). Sob o ponto de vista funcional, outras hipóteses podem ser levantadas a partir da questão da dinapenia. É possível que a fraqueza muscular do idoso conduza a um declínio na funcionalidade, o que naturalmente reduz os níveis de atividade física e algumas vezes pode provocar quadros de imobilizações (GOODPASTER et al., 2006). Estas condições podem consequentemente levar a uma secundária atrofia muscular por desuso.

Todos estes mecanismos, de ordem micro ou macroscópica, conduzem o músculo esquelético do idoso a apresentar uma redução da sua capacidade de produção de força, geral ou mesmo por unidade contrátil. Dessa forma, tornam-se evidentes as justificativas pelas quais o envelhecimento afeta, por si só, negativamente a funcionalidade e a mobilidade do sujeito submetido a este processo.

\section{Diagnóstico da sarcopenia e da dinapenia}

Dado o crescente número de investigações nesta área de interesse, nos últimos anos, algumas entidades internacionais têm se esforçado para desenvolver critérios diagnósticos para identificar e classificar os casos de sarcopenia e dinapenia. Nesse contexto, o Grupo Europeu de Trabalho em Sarcopenia com o Idoso (CRUZ-JENTOFT et al., 2010) sugere um algoritmo de sarcopenia, que utiliza os seguintes componentes para seu diagnóstico: idade, velocidade de 
marcha, força de preensão e massa muscular. De acordo com este algoritmo, todos os idosos com idade superior a 65 anos devem ser avaliados quanto a sua velocidade de marcha. Aqueles que apresentarem valores iguais ou abaixo de $0,80 \mathrm{~m} / \mathrm{s}$ devem ser testados quanto à sua massa muscular total ou apendicular, que será usada para o diagnóstico da sarcopenia. Por outro lado, aqueles idosos que apresentarem valores de velocidade de marcha superiores a $0,80 \mathrm{~m} / \mathrm{s}$ deverão realizar o teste de preensão manual. Idosos com baixos valores de preensão manual são solicitados a realizar a avaliação de massa muscular total ou apendicular para diagnóstico de sarcopenia. Os demais são considerados não-sarcopênicos.

Em 2012, com uma abordagem voltada especificamente para a dinapenia, um novo algoritmo foi sugerido (MANINI; CLARK, 2012), levando em consideração condições biológicas, psicológicas, de meio ambiente e de origens sociológicas como potentes fatores que influenciam para a criação de fatores de risco para a função física. Nesse algoritmo, indivíduos acima de 60 anos devem ser avaliados quanto a seus fatores de risco. Aqueles que apresentarem altos fatores de risco para o desenvolvimento de dinapenia deverão realizar o teste de força de extensão de joelhos, que resultará no diagnóstico positivo ou negativo de dinapenia. Por outro lado, aqueles que apresentarem baixo ou nenhum risco, deverão realizar um teste de preensão manual. Aqueles que demonstrarem baixa força de preensão manual serão solicitados a realizar o teste de força de extensão de joelhos para obtenção do diagnóstico e aqueles que apresentarem resultados satisfatórios na preensão manual, terão o diagnóstico negativo para dinapenia. Um fluxograma de ações de ambos os algoritmos é apresentado na Figura 1. 
Figura 1 - Fluxograma de ações do algoritmo criado pelo Grupo Europeu de Trabalho em Sarcopenia com o Idoso (A) e do algoritmo proposto por Manini e Clark (B)
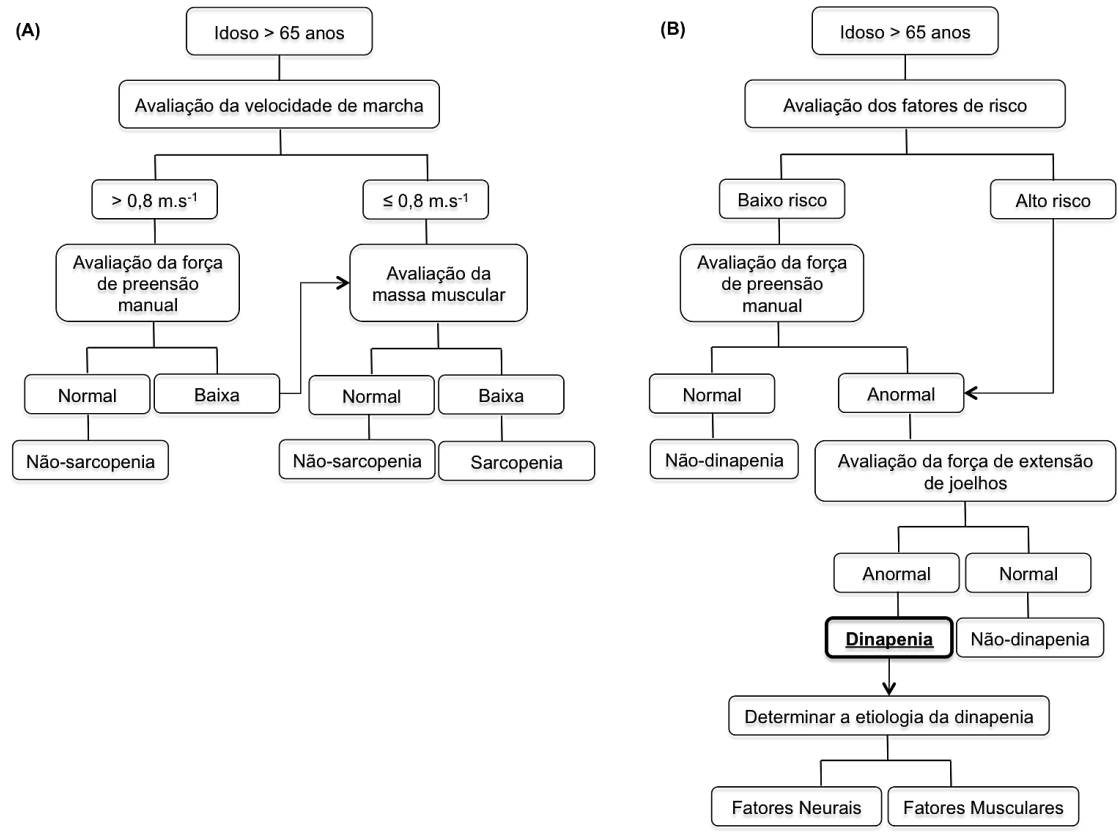

Fonte: Elaborada pelos autores com base em Cruz-Jentoft et al. (2010) e Manini e Clark (2012).

É possível identificar pontos fortes e fracos em ambos os algoritmos. Dentre eles pode-se destacar: a utilização de testes como o de velocidade de marcha e o de preensão manual viabiliza sua aplicação em diversos contextos de prática clínica com idosos. Ainda, o equipamento específico para realização do teste de extensão de joelhos é usualmente encontrado em ambientes de treinamento, no entanto, há uma grande dificuldade de comparação destes valores devido às diferenças nos equipamentos. Além disto, a avaliação dos fatores de risco para dinapenia, quanto às classificações alto risco ou baixo risco é subjetiva, dependendo do avaliador escolher qual decisão tomar para encaminhar o idoso ao próximo passo do algoritmo. Em ambos os modelos, o nível de força exigido para a classificação de dinapenia/sarcopenia ainda é subjetivo, visto que o ponto de corte para força muscular adequada tanto de membros superiores quanto de membros inferiores no idoso ainda não está estabelecido na literatura (MANINI; CLARK, 2012). 
A fim de amenizar os efeitos dos processos de dinapenia e sarcopenia, o treinamento de força tem sido recomendado (BECKWÉE et al., 2019; LAW et al., 2016). De fato, uma vasta gama de revisões sistemáticas com metanálises já demonstraram que o treinamento de força é efetivo para melhorar a força e a massa muscular de homens e mulheres idosos (BORDE; HORTOBÁGYI; GRANACHER, 2015; BUCH et al., 2017; CSAPO; ALEGRE, 2016; GUIZELINI et al., 2018; LATHAM et al., 2004; PETERSON et al., 2010; PETERSON; SEN; GORDON, 2011; STEIB; SCHOENE; PFEIFER, 2010; STRAIGHT et al., 2016; YOSHIMURA et al., 2017).

Em uma metanálise avaliando mais de 1070 homens e mulheres com idades entre 50 e 92 anos, Peterson et al. (2010) verificaram que o treinamento de força aumenta a força máxima de membros inferiores $(29 \pm 2 \%$ e $33 \pm 3 \%$ para a força avaliada nos exercícios leg press e extensão de joelhos, respectivamente) e superiores ( $24 \pm 2 \%$ e $25 \pm 2 \%$ para a força avaliada nos exercícios supino e puxada, respectivamente). Esses incrementos foram observados após intervenções com duração entre 6 a 52 semanas (duração média de 17,6 $6 \pm 8,6$ semanas) realizados de uma a três vezes por semana (média de $2,7 \pm 0,5$ vezes por semana). Cabe salientar que a adaptação da força muscular ao treinamento de força foi similar entre homens e mulheres e entre diferentes décadas de vida, sugerindo que homens e mulheres possuem a mesma capacidade de ganhar força máxima dos 50 aos 90 anos.

A magnitude de aumento da força máxima advinda do treinamento de força é dependente de variáveis do treinamento, como intensidade, volume e frequência semanal. Parece existir consenso que maiores intensidades de treinamento promovem maiores incrementos (CSAPO; ALEGRE, 2016; PETERSON et al., 2010; STEIB; SCHOENE; PFEIFER, 2010). Já em relação ao volume de treinamento, as evidências são divergentes. Enquanto que na metanálise de Steib, Schoene e Pfeifer (2010) observou-se que uma série por exercício promoveu os mesmos incrementos de força do que três séries, na metanálise de Borde, Hortobágyi e Granacher (2015), avaliando a relação dose-resposta do treinamento de força, verificou-se que duas a três séries por exercício é o volume ideal para promover ganhos de força máxima. Por fim, os ganhos de força máxima são maximizados quando duas sessões de treinamento por semana são realizadas.

Além de estimular ganhos na força máxima, o treinamento de força também proporciona incremento da força potente. Esse é um fato importante 
porque a potência muscular é fortemente associada, inclusive mais do que a força máxima, à capacidade do idoso de realizar suas atividades de vida diária e ao risco de quedas (BEAN et al., 2002). Estudos têm demonstrado que tanto o treinamento de força tradicional quanto o treinamento de potência (no qual realiza-se a fase concêntrica do movimento o mais rápido possível) melhoram esse desfecho, entretanto, os ganhos são superiores quando o treinamento de potência é realizado (STEIB; SCHOENE; PFEIFER, 2010; STRAIGHT et al., 2016). Aumentos de $21,5 \%$ a 33,4\% na potência muscular foram observados após 6 a 24 semanas de treinamento de potência realizado de duas a três vezes por semana (duas a quatro séries por exercício) (KATSOULIS; STATHOKOSTAS; AMARA, 2019). Katsoulis, Stathokostas e Amara (2019) verificaram que o treinamento de potência utilizando intensidades de 20 a 80\% de 1RM (uma repetição máxima) promoveram melhora da potência muscular em idosos, mas altas ( $<70 \%$ de $1 \mathrm{RM})$ e moderadas intensidades (51-69\% de 1RM) induziram maiores aumentos quando comparadas com baixa intensidade $(<50 \%$ de 1RM). Em relação ao volume do treinamento de potência, uma série por exercício promoveu os mesmos ganhos de força máxima e de força potente em comparação a três séries em mulheres idosas previamente sedentárias (RADAELLI et al., 2018).

O aumento da força muscular em decorrência de um treinamento de força pode ser explicado por adaptações em fatores musculares e neurais. É consenso que o treinamento de força promove incrementos na massa muscular de homens e mulheres idosos (BORDE, HORTOBÁGYI; GRANACHER, 2015; CSAPO; ALEGRE, 2016; PETERSON; SEN; GORDON, 2011). A adaptação na massa muscular é influenciada por fatores relacionados ao treinamento e à idade dos participantes. Um maior volume de treinamento (número de séries) foi associado a um maior aumento da massa magra (PETERSON; SEN; GORDON, 2011) e sugere-se que duas a três séries é o volume ideal para maximizar os ganhos de massa muscular (BORDE, HORTOBÁGYI; GRANACHER, 2015). Além disso, três sessões semanais é a frequência mais indicada para promover ganhos de massa muscular (BORDE, HORTOBÁGYI; GRANACHER, 2015). Em relação à intensidade do treinamento, parece que maiores cargas promovem maiores incrementos (CSAPO; ALEGRE, 2016). Por fim, quanto maior a idade do participante, menores os ganhos advindos do treinamento de força na massa muscular (PETERSON; SEN; GORDON, 2011). Além do aumento da massa muscular, outros fatores musculares como o aumento da rigidez tendínea (ERIKSEN et al., 2019; MAGANARIS; NARICI; REEVES, 2004) e do ângulo de penação (REEVES; NARICI; MAGANARIS, 2004) são responsáveis pelo aumento da força muscular após um período de intervenção com exercícios de força. 
Adaptações neurais como o aumento da atividade neural agonista e a diminuição da atividade neural antagonista (GRANACHER; GRUBER; GOLLHOFER, 2009; HÄKKINEN et al., 2001) têm sido relatadas após treinamentos de força em idosos e ajudam a explicar os aumentos de força muscular. Além disso, também já se registrou aumento da máxima taxa de frequência de disparo das unidades motoras (KAMEN; KNIGHT, 2004) e aumento do drive eferente (UNHJEM et al., 2015).

As evidências apontam para um efeito benéfico do treinamento de força na força máxima, na força potente e na massa muscular em idosos, sendo que esses benefícios já são observados após um curto período de intervenção (6 a 8 semanas). O incremento da força muscular pode ser explicado por adaptações musculares e neurais que se encontram deterioradas pelo processo de envelhecimento.

\section{Conclusões}

Esta revisão narrativa demonstrou que a dinapenia, apesar de ser um conceito recente trata de uma área de conhecimento em crescimento na literatura científica atual. Pode-se, por meio dos dados apresentados, concluir que a contribuição de explicação advinda da massa muscular para a capacidade de produção de força no envelhecimento é muito pequena (em torno de 5\%), podendo-se atribuir a fatores neurais e musculares a maior parte da explicação dos mecanismos pelos quais a dinapenia se instala nos idosos. Quanto aos fatores musculares descritos, observou-se que são inúmeros os achados recentes da literatura. Sendo o seu conhecimento de extrema importância para o embasamento dos pesquisadores e profissionais da saúde visando uma prescrição otimizada de tratamentos preventivos e de reabilitação idosos com dinapenia.

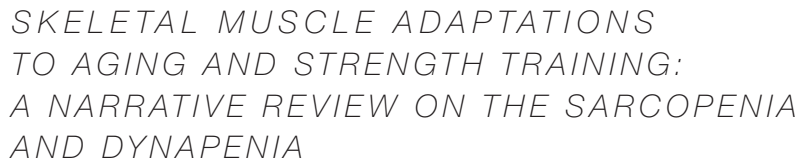

The growth of the older adult population underscores the importance of research about effects of the aging in the skeletal system. The aim of this study was to discuss about the sarcopenia and dynapenia terminology, as to revise the skeletal muscle adaptations to aging and 
to strength training. The electronic search was conducted in MEDLINE and Scopus in March 2016 using the terms aging, skeletal muscle, strength and dynapenia. The decline of muscle strength production capacity as a result of aging received the scientific name dynapenia. Despite this, still exists a range of researchers who uses this definition as part of the "Sarcopenia" construct. Neural and muscular factors constitute the mechanisms that contribute to the installation of the dynapenia. Neural factors include changes in recruitment and synchronization of motor units, drop in firing rate and nerve conduction velocity, denervation of type II muscle fibers (fast) by neuronal death and reinnervation of type I fibers (slow). Muscular factors consist of changes in muscle architecture, fiber type changes, dysfunctions in the excitation-contraction coupling process, myosin structural changes and intra and inter muscular lipid infiltration. The dinapenia is diagnosed based on an assessment of risk factors, the knee extension strength and handgrip test. From the understanding of the various mechanisms that contribute to the installation of dinapenia, health sciences researchers may have expertise for better prescribing preventive treatments or even dinapenia rehabilitation.

keywords

Skeletal Muscle. Aging. Muscle Strength. Muscle Weakness.

referências

ALEXANDRE, Tiago da Silva et al. Sarcopenia according to the european working group on sarcopenia in older people (EWGSOP) versus dynapenia as a risk factor for mortality in the elderly. The Journal of Nutrition Health and Aging, New York, v. 18, n. 8, p. 751-756, 2014.

ANDERSON, Ayuk Agbor et al. The novel skeletal muscle sarcoplasmic reticulum JP-45 protein: molecular cloning, tissue distribution, developmental expression, and interaction with alpha 1.1 subunit of the voltage-gated calcium channel. The Journal of Biological Chemistry, Baltimore, v. 278, n. 41, p. 39987-39992, Oct. 2003.

ANDERSON, Ayuk Agbor et al. The junctional sr protein JP-45 affects the functional expression of the voltage-dependent Ca2+ channel cav1.1. Journal of Cell Science, London, v. 119, n. 10, p. 2145-2155, May 2006.

BATISTA, Rafael Reimann; VAZ, Marco Aurélio. Arquitetura muscular e envelhecimento: adaptação funcional e aspectos clínicos: revisão da literatura. Fisioterapia e Pesquisa, São Paulo, v. 16, n. 4, p. 368-373, out./dez. 2009.

BAUTMANS, Ivan et al. Circulating acute phase mediators and skeletal muscle performance in hospitalized geriatric patients. The Journals of Gerontology: Series A, Biological Sciences and Medical Sciences, Washington, v. 60, n. 3, p. 361-367, Mar. 2005. 
BEAN, Jonathan et al. The relationship between leg power and physical performance in mobility limited older people. Journal of the American Geriatrics Society, New York, v. 50, n. 3, p. 461-467, Mar. 2002.

BECKWÉE, David et al. Exercise interventions for the prevention and treatment of sarcopenia. a systematic umbrella review. Journal of Nutrition, Health and Aging, New York, v. 23, n. 6, p 494-502, Jan. 2019.

BORDE, Ron; HORTOBÁGYI, Tibor; GRANACHER, Urs. Dose-response relationships of resistance training in healthy old adults: a systematic review and meta-analysis. Sports Medicine, Auckland, v. 45, n. 12, p. 1693-1720, Dec. 2015.

$\mathrm{BUCH}$, Assaf et al. Circuit resistance training is an effective means to enhance muscle strength in older and middle aged adults: a systematic review and meta-analysis. Ageing Research Reviews, Oxford, v. 37, p. 16-27, Aug. 2017.

CLARK, Brian. Neuromuscular changes with aging and sarcopenia. The Journal of Frailty \& Aging, Toulouse, v. 8, n. 1, p. 7-9, Jan. 2019.

CLARK, Brian; MANINI, Todd. Sarcopenia $\neq$ dynapenia. Journal of Gerontology, Washington, v. 63, n. 8, p. 829-834, Aug. 2008

CLARK, Brian et al. Adaptations in human neuromuscular function following prolonged unweighting: II neurological properties and motor imagery efficacy. Journal of Applied Physiology, Washington, v. 101, n. 1, p. 264-272, July 2006.

CRUZ-JENTOFT, Afonso et al. Sarcopenia: european consensus on definition and diagnosis: report of the european working group on sarcopenia in older people. Age and Ageing, London, v. 39, n. 4, p. 412-423, July 2010

CSAPO, Robert; ALEGRE, Luis. Effects of resistance training with moderate vs heavy loads on muscle mass and strength in the elderly: a meta-analysis. Scandinavian Journal of Medicine \& Science in Sports, Copenhagen, v. 26, n. 9, p. 995-1006, Sep. 2016

DELBONO, Osvaldo. Expression and regulation of excitation-contraction coupling proteins in aging skeletal muscle. Current Aging Science, Indianapolis, v. 4, n. 3, p. 248-259, 2011.

DELBONO, Osvaldo et al. Loss of skeletal muscle strength by ablation of the sarcoplasmic reticulum protein JP45. Proceedings of the National Academy of Sciences of the United States of America, Washington, v. 11, n. 104, p. 20108-20113, Dec. 2007.

DIMITRU, Adrian et al. Muscle changes during atrophy. In: XIAO, Junjie. Muscle atrophy. Singapore: Springer Singapore, 2018. p. 73-92

DRAGANIDIS, Dimitrios et al. Inflammaging and skeletal muscle: can protein intake make a difference? The Journal of Nutrition, Maryland, v. 146, n. 10, p. 1940-1952, Oct. 2016.

ERIKSEN, Christian et al. Load magnitude affects patellar tendon mechanical properties but not collagen or collagen cross-linking after long-term strength training in older adults. BMC Geriatrics, London, v. 19, n. 1, p. 30, Jan. 2019

EVANS, William. What is sarcopenia? The Journals of Gerontology: Series A, Biological Sciences and Medical Sciences, Washington, v. 50, n. Spec. 5-8, Nov. 1995.

FRONTERA, Walter et al. A cross-sectional study of muscle strength and mass in 45 to 78-year-old men and women. Journal of Applied Physiology, Washington, v. 71, n. 2, p. 644-650, Aug. 1991.

GOODPASTER, Bret et al. Attenuation of skeletal muscle and strength in the elderly: The Health ABC Study. Journal of Applied Physiology, Washington, v. 90, n. 6, p. 2157-2165, June 2001 . 
GOODPASTER, Bret et al. Skeletal muscle attenuation determined by computed tomography is associated with skeletal muscle lipid content. Journal of Applied Physiology, Washington, v. 89, n. 1, p. 104-110, July 2000.

GOODPASTER, Bret et al. The loss of skeletal muscle strength, mass, and quality in older adults: The Health, Aging and Body Composition Study. The Journals of Gerontology: Series A, Biological Sciences and Medical Sciences, Washington, v. 61, n. 10, p. 1059-1064, Oct. 2006.

GRANACHER, Urs; GRUBER, Markus; GOLLHOFER, Albert. Resistance training and neuromuscular performance in seniors. International Journal of Sports Medicine, Stuttgart, v. 30, n. 9, p. 652-657, Sep. 2009.

GUIZELINI, Pedro de Camargo et al. Effect of resistance training on muscle strength and rate of force development in healthy older adults: a systematic review and meta-analysis. Experimental Gerontology, Oxford, v. 102, p. 51-58, Feb. 2018.

HÄKKINEN, Keijo et al. Changes in electromyographic activity, muscle fiber and force production characteristics during heavy resistance/power strength training in middle-aged and older men and women. Acta Physiologica Scandinavica, Stockholm, v. 171, n. 1, p. 51-62, Jan. 2001.

HUGHES, Virginia et al. Longitudinal muscle strength changes in older adults: influence of muscle mass, physical activity, and health. The Journals of Gerontology: Series A, Biological Sciences and Medical Sciences, Washington, v. 56, n. 5, p. B209-217, May 2001.

HUNTER, Sandra; PEREIRA, Hugo; KEENAN, Kevin. The aging neuromuscular system and motor performance. Journal of Applied Physiology, Washington, v. 121, p. 982-995, Aug. 2016

INSTITUTO BRASILEIRO DE GEOGRAFIA E ESTATÍSTICA (IBGE). Censo 2010. Rio de Janeiro: IBGE, 2010. Disponível em: www.censo2010.ibge.gov.br. Acesso em: 8 jul. 2019.

JIMENEZ-MORENO, Ramón et al. Sarcoplasmic reticulum Ca2+ release declines in muscle fibers from aging mice. Biophysical Journal, New York, v. 94, n. 8, p. 3178-3188, Apr. 2008.

KAMEN, Gary; KNIGHT, Christopher. Training-related adaptations in motor unit discharge rate in young and older adults. The Journals of Gerontology: Series A, Biological Sciences and Medical Sciences, Washington, v. 59, n. 12, p. 1334-1338, Dec. 2004.

KARAMANIDIS, Kiros; ARAMPATZIS, Adamantios. Mechanical and morphological properties of human quadriceps femoris and triceps surae muscle-tendon unit in relation to aging and running. Journal of Biomechanics, Elmsford, v. 39, n. 3, p. 406-417, 2006.

KATSOULIS, Konstantina; STATHOKOSTAS, Liza; AMARA, Catherine. The effects of high- versus low-intensity power training on muscle power outcomes in healthy, older adults: a systematic review. Journal of Aging and Physical Activity, Sascatchewan, v. 27, n. 3, p. 422-439, June 2019.

$\mathrm{KUBO}$, Keitaro et al. Muscle architectural characteristics in young and elderly men and women. International Journal of Sports Medicine, Stuttgart, v. 24, n. 2, p. 125-130, Feb. 2003.

LATHAM, Nancy et al. Systematic review of progressive resistance strength training in older adults. The Journals of Gerontology: Series A, Biological Sciences and Medical Sciences, Washington, v. 59A, n. 1, p. 48-61, Jan. 2004.

LAURETANI, Fulvio et al. Axonal degeneration affects muscle density in older men and women. Neurobiology of Aging, New York, v. 27, n. 8, p. 1145-1154, Aug. 2006.

LAW, Timothy et al. Resistance exercise to prevent and manage sarcopenia and dynapenia. Annual Review of Gerontology and Geriatrics, New York, v. 36, n. 1, p. 205-228, Dec. 2016. 
LIEBER, Richard; FRIDÉN, Jan. Clinical significance of skeletal muscle architecture. Clinical Orthopaedics and Related Research, Philadelphia, v. 383, p. 140-151, Feb. 2001.

LOWE, Dawn; THOMAS, David; THOMPSON, LaDora. Force generation, but not myosin atpase activity, declines with age in rat muscle fibers. American Journal of Physiology: Cell Physiology, Bethesda, v. 283, n. 1, p. C187-C192, July 2002.

MACINTOSH, Brian; GARDINER, Phillip; MCCOMAS, Allan. Skeletal muscle: form and function. 2. ed. Champaign, IL: Human Kinetics, 2006

MAGANARIS, Costis; NARICI, Marco; REEVES, Neil. In vivo human tendon mechanical properties: effect of resistance training in old age. Journal of Musculoskeletal and Neuronal Interactions, Kifissia, v. 4, n. 2, p. 204-208, June 2004.

MANINI, Todd; CLARK, Brian. Dynapenia and aging: an update. The Journals of Gerontology: Series A, Biological Sciences and Medical Sciences, Washington, v. 67, n. 1, p. 28-40, Jan. 2012

MAUGHAN, Ron; WATSON, Jeniffer; WEIR, John. Strength and cross-sectional area of human skeletal muscle. The Journal of Physiology, London, v. 338, p. 37-49, May 1983.

NARICI, Marco; MAFFULLI, Nicola. Sarcopenia: characteristics, mechanisms and functional significance. British Medical Bulletin, London, v. 95, n. 1, p. 139-159, Sep. 2010.

NARICI, Marco; MAGANARIS, Constantinos. Adaptability of elderly human muscles and tendons to increased loading. Journal of Anatomy, London, v. 208, n. 4, p. 433-443, Apr. 2006

NARICl, Marco et al. Effect of aging on human muscle architecture. Journal of Applied Physiology, Washington, v. 95, n. 6, p. 2229-2234, Dec. 2003.

NEWMAN, Anne et al. Strength, but not muscle mass, is associated with mortality in the health, aging and body composition study cohort. The Journals of Gerontology: Series A, Biological Sciences and Medical Sciences, Washington, v. 61, n. 1, p. 72-77, Jan. 2006.

ORGANIZAÇÃO MUNDIAL DE SAÚDE (OMS). Brasil. Genebra: OMS, 2015. Disponível em: http://www.who.int/countries/bra/es. Acesso em: 8 jul. 2019.

PETERSON, Mark; SEN, Ananda; GORDON, Paul. Influence of resistance exercise on lean body mass in aging adults: a meta-analysis. Medicine \& Science in Sports \& Exercise, Madison, v. 43, n. 2, p. 249-258, Feb. 2011.

PETERSON, Mark et al. Resistance exercise for muscular strength in older adults: A meta-analysis. Ageing Research Reviews, Oxford, v. 9, n. 3, p. 226-237, July 2010.

RADAELLI, Régis et al. Higher muscle power training volume is not determinant for the magnitude of neuromuscular improvements in elderly women. Experimental Gerontology, Oxford, v. 110, p. 15-22, Sep. 2018.

REEVES, Neil; NARICI, Marco; MAGANARIS, Costis. In vivo human muscle structure and function: adaptations to resistance training in old age. Experimental Physiology, Cambridge, v. 89, n. 6, p. 675-689, Nov. 2004.

ROSENBERG, Irwin Harold. Summary comments. The American Journal of Clinical Nutrition, Bethesda, v. 50, p. 1231-1233, 1989.

SONG, Mi Yeon et al. Sarcopenia and increased adipose tissue infiltration of muscle in elderly African American women. The American Journal of Clinical Nutrition, Bethesda, v. 79, n. 5, p. $874-880$, May 2004

STEIB, Simon; SCHOENE, Daniel; PFEIFER, Klaus. Dose-response relationship of resistance training in older adults: a meta-analysis. Medicine \& Science in Sports \& Exercise, Madison, v. 42, n. 5, p. 902-914, May 2010. 
STRAIGHT, Chad et al. Effects of resistance training on lower-extremity muscle power in middle-aged and older adults: a systematic review and meta-analysis of randomized controlled trials. Sports Medicine, Auckland, v. 46, n. 3, p. 353-364, Mar. 2016.

TIELAND, Michael; TROUWBORST, Inez; CLARK, Brian. Skeletal muscle performance and ageing. Journal of Cachexia, Sarcopenia and Muscle, Berlin, v. 9, n. 3, p. 3-19, Feb. 2018.

UNHJEM, Runar et al. Strength training-induced responses in older adults: attenuation of descending neural drive with age. Age (Dordr), Grandville, v. 37, n. 3, p. 9784, June 2015

VISSER, Marjolein et al. Muscle mass, muscle strength, and muscle fat infiltration as predictors of incident mobility limitations in well-functioning older persons. The Journals of Gerontology: Series A, Biological Sciences and Medical Sciences, Washington, v. 60, n. 3, p. 324-333, Mar. 2005

WELLE, Stephen; BHATT, Kirti; THORNTON, Charles. High-abundance mRNAs in human muscle: comparison between young and old. Journal of Applied Physiology, Washington, v. 89, n. 1, p. 297-304, July 2000

YOSHIMURA, Yoshihiro et al. Interventions for treating sarcopenia: a systematic review and meta-analysis of randomized controlled studies. Journal of the American Medical Directors Association, Hagerstown, v. 18, n. 6, p. 553.e1-553.e16, June 2017.

Data de Submissão: 20/09/2017

Data de Aprovação: 22/08/2019 
